Original Article

\title{
INVESTIGATING THE POTENTIAL OF AN ANTIDEPRESSANT INTRANASAL MUCOADHESIVE MICROEMULSION
}

\author{
GURPREET SINGH*, NISHA RAWAT, KIRTI SINGH, AMITA SARWAL, V. R. SINHA \\ 1University Institute of Pharmaceutical Sciences, Panjab University, Chandigarh \\ Email: pugurpreet@gmail.com \\ Received: 10 Mar 2018 Revised and Accepted: 08 May 2018
}

\begin{abstract}
Objective: The main aim of this study was to formulate, develop and optimized a duloxetine hydrochloride (dlx-hcl) loaded mucoadhesive microemulsion intended for intranasal administration.

Methods: Established on solubility studies capmul mcm, transcutol-p, labrasol were used as oil, co-surfactant and surfactant respectively. The optimized mucoadhesive microemulsion prepared using water titration method was further characterized for particle size, polydispersity index, zeta potential and conductivity measurements followed by drug content, nasal cilio toxicity and biochemical estimation of the selected formulation.

Results: All physicochemical parameters conducted, proved that dlx-hcl microemulsion was appropriate for nasal delivery. Chitosan, used as mucoadhesive polymer demonstrated enhanced retention time of the microemulsion in nasal mucosa with no signs of toxicity and epithelial damage. The particle size and zeta potential were found to be of $200 \mathrm{~nm}$ and-15 $\mathrm{mV}$ respectively considering the formulation safe for nasal delivery.
\end{abstract}

Conclusion: This formulation strategy can be used as an effective targeting technique for the drugs having low bioavailability and poor brain penetration along with an effective method for the treatment long-term disease like depression.

Keywords: Blood-brain barrier, Nasal mucosa, Intranasal delivery, Microemulsion, Mucoadhesive, Duloxetine hydrochloride

(C) 2018 The Authors. Published by Innovare Academic Sciences Pvt Ltd. This is an open access article under the CC BY license (http://creativecommons.org/licenses/by/4.0/] DOI: http://dx.doi.org/10.22159/ijpps.2018v10i6.25710

\section{INTRODUCTION}

Treatment involving site-specific delivery to the central nervous system along with prolonged presence and sustain release of a drug to attain steady-state levels is regarded as an ideal effective psychotherapy. Major obstacles to this pathway include the bloodbrain barrier (bbb) prohibiting the entry of the drug into the brain $[1,2]$. Intranasal delivery has become prominent as an alternative to invasive delivery systems in order to bypass the bbb and facilitate targeted therapeutic delivery. This delivery system utilizes olfactory and trigeminal nerve pathways [3]. The nasal route possesses advantages of rapid absorption of the drug, higher bioavailability allowing lower doses, faster onset of action [4,5]. Nasal route protects drugs and biomolecules from susceptible enzymatic or acidic degradation, can deliver drugs directly to the brain via olfactory lobe and eliminate first pass metabolism [6]. Though usage of the nasal route in human medicine has been mostly limited to locally acting agents, in recent years a few systemically acting nasal drug products have been commercialized for menopausal symptoms, pain control, endometriosis and migraine headache [7].

Microemulsions (MEs) as colloidal carriers within size range are clear, optically isotropic and thermodynamically stable systems consisting of oil, water and surfactants with/or a co-surfactant [8]. Additionally, incorporation of a mucoadhesive agent in the microemulsion increases the nasal residence time, overcoming the mucociliary clearance (mcc) and enhancing bioavailability [9].

Microemulsion-based delivery systems hold numerous characteristics like thermodynamic stability, increased drug loading, enhanced penetration through the biological membranes, increased bioavailability and less inter and intra-individual variability in drug pharmacokinetics making them suitable for intranasal drug delivery [10, 11]. Microemulsions have very low surface tension and small droplet size which results in high absorption and permeation and can cross the blood-brain barrier due to their stable nature $[12,13]$.

Constraints of nasal administration include natural defence mechanism: nasal mucociliary clearance. Therefore, it is requisite for localization of the formulation on the mucosal layer of nasal cavity for enhanced drug absorption along with optimum retention. The inclusion of a well-designed mucoadhesive agent to microemulsion can intercept rapid nasal clearance of formulation $[14,15]$. Duloxetine hydrochloride (dlx-hcl) $\{(\mathrm{b})-(\mathrm{S})-\mathrm{N}-$ methyl-g-(1-naphthalenyloxy)-2thiophene propanamine hydrochloride is a potent, balanced dual reuptake inhibitor of both serotonin and norepinephrine (snri) [16] recommended for maintenance treatment of the major depressive disorder. It undergoes hepatic first-pass metabolism, exhibits average oral bioavailability of $50 \%$ (systemic), is acid labile at gastric $\mathrm{pH}(\mathrm{pH} \mathrm{1-2)}$ and has a low cerebrospinal fluid concentration on oral administration $[17,18]$.

Effective treatment using antidepressants relies upon carrier systems exploiting approaches for drug delivery to the brain with a continued prolonged presence in the brain [19]. This study was aimed at developing a stable mucoadhesive dlx-hcl microemulsion for intranasal administration to improve the bioavailability of this drug and provide high drug brain levels along with formulation and characterization of dlx-hcl microemulsion in order to explore the feasibility of this route of administration.

\section{MATERIALS AND METHODS}

\section{Materials}

Drugs and reagent: Duloxetine hydrochloride was gifted by Shodhana Laboratories Limited Laboratories (Hyderabad, India). Capmul mcm was obtained as a gift sample from Abitec Corporation (Janesville, United States), labrafac, labrafil 1944, labrafil 2125, transcutol p, labrasol, cremphore, were received as gift sample by Gattefosse Asia Ltd. (Mumbai, India) pluronic F-127, Pluronic F-68 and PEG 300, PEG 400, thiobarbituric acid were purchased from Sigma Aldrich Chemical private Ltd, Bangalore, India. Tween 80, span 80, oleic acid, potassium dihydrogen phosphate, methanol and propylene glycol were obtained from S. D Fine Chemicals, Mumbai, India. Sodium chloride and Copper sulphate were obtained from Loba Chemie, Mumbai, India. All other ingredients used in the study were of analytical grade. 


\section{Methods}

\section{Solubility studies}

The solubility of duloxetine hydrochloride in different oils (labrafil M 1944, labrafil M 2125, capmul mcm) surfactants (tween 80, labrasol, span 80) and co-surfactants (transcutol P, PEG 300, PEG 400, chemophore RH 40) were determined. The drug was added in excess to the excipients in a tightly closed eppendorf tube and was mixed thoroughly using vortex mixture. The dispersions were kept at $37{ }^{\circ} \mathrm{C}$ under agitation in water bath shaker for $72 \mathrm{~h}$ for equilibration. After $72 \mathrm{~h}$ of equilibration, they were centrifuged at 3000 revolutions per minute (rpm) for $15 \mathrm{~min}$ and the supernatant was then filtered and diluted with an appropriate solvent (methanol) for analysis. The analysis was done using uv spectroscopy at $\lambda \max$ of $230 \mathrm{~nm}[20]$.

\section{Pseudoternary phase diagram}

The pseudoternary phase diagram of oil, surfactant, co-surfactant and water were constructed using a water titration method. The surfactant was blended with co-surfactant in fixed weight ratios $(1: 1,2: 1)$ of labrasol and transcutol p. Different combinations of oil and $s_{\text {mix }}$ were made so that the maximum ratios were covered in order to precisely delineate the boundaries of phases formed in the phase diagrams. For each phase diagram, the ratio of oil to the $\mathbf{s}_{\text {mix }}$ was varied as $9: 1,8: 2,7: 3,6: 4,5: 5,4: 6,3: 7,2: 8,1: 9(\mathrm{w} / \mathrm{w})$. Phase diagram was constructed using Triplot software version 4.1.2. The mixture of oil and $s_{\text {mix }}$ at certain weight ratios were diluted with water dropwise under moderate stirring with a magnetic stirrer. After $24 \mathrm{~h}$ of stirring, the mixtures were assessed visually and determined as being a clear and transparent microemulsion.

\section{Thermodynamic stability studies}

The selected formulation was assessed for thermodynamic stability by means of visual clarity and phase separation by applying stress tests, including centrifugation and freeze-thaw cycles.

Heating cooling cycle: Six cycles at refrigerator temperatures between $4^{\circ} \mathrm{C}$ and $45^{\circ} \mathrm{C}$ with storage for not less than $48 \mathrm{~h}$ were conducted, and the formulation examined for stability.

Centrifugation test: Formulations were centrifuged at $3500 \mathrm{rpm}$ for $30 \mathrm{~min}$ and observed for phase separation.

Freeze-thaw cycle: Thee freeze-thaw cycles were performed at between -21 and $+25^{\circ} \mathrm{C}$, for not less than $48 \mathrm{~h}$ at each temperature.

\section{Optimization of mucoadhesive and gelling excipients}

Various mucoadhesive and gelling excipients were screened to ascertain thermoresponsive behaviour of gelling and mucoadhesive excipients. Pluronic F127 and pluronic F68 were screened as gelling agents while chitosan and carbopol $934 \mathrm{P}$ were screened as mucoadhesive agents. Thermoreversible gels of pluronic F127 and pluronic F68 were prepared using a cold method by dissolving the pluronic in distilled water and phosphate buffer (pH 6.7 and 7.4), at $4{ }^{\circ} \mathrm{C}$. The solution was kept at $4{ }^{\circ} \mathrm{C}$ until clear and then gradually increased from 15 to $40{ }^{\circ} \mathrm{C}$ to visually assess its gelling behavior. Similarly, dispersions of the mucoadhesive agent in various concentrations were prepared, weighed, soaked in water and kept aside overnight at room temperatures to visually examine its gelling behavior.

\section{Preparation of formulation}

Dlx-hcl microemulsion (ME) was prepared by the water titration method. The calculated amount of drug was added to the oily phase of ME and magnetically stirred until dissolved, followed by addition of surfactant-cosurfactant $\left(\mathrm{s}_{\mathrm{mix}}\right)$ mixture to produce a clear mixture. Mucoadhesive and gelling excipients were dissolved in the aqueous phase and kept at $4{ }^{\circ} \mathrm{C}$ to obtain a clear solution. The final drug-oil$\mathrm{s}_{\text {mix }}$ mixture was then titrated with the aqueous phase to obtain mucoadhesive microemulsion and stored at refrigerated temperature $\left(4^{\circ} \mathrm{C}\right)$ for further studies. Entrapped bubbles were removed by placing the gels in vacuum desiccators.

\section{Preparation of drug solution}

The dlx-hcl solution meant for comparative evaluation of ME-based systems was prepared by dissolving dlx-hcl $(50 \mathrm{mg})$ in $10 \mathrm{ml}$ of methanol resulting in a solution of $5 \mathrm{mg} / \mathrm{ml}$.

\section{Characterization of formulation}

\section{Microemulsion}

\section{Particle size analysis}

Particle sizes of the formulations were evaluated using zetasizer; it was equipped with He-Ne laser source operating at a wavelength of $633 \mathrm{~nm}$. Viscosity was kept constant, with a material refractive index of $1.330 .1 \mathrm{ml}$ of sample was taken into a cuvette and placed in the instrument for sample analysis.

\section{Zeta potential}

The measurement of zeta potential has become completely connected with the characterization of colloidal dispersions as this parameter is highly useful for the assessment of the physical stability of colloidal dispersions. Zeta potential was measured by using Beckman Coulter Delsa ${ }^{\mathrm{TM}}$ nano.

\section{Viscosity}

The viscosity of samples was measured at $37^{\circ} \mathrm{C}^{*}$ the help of rotational viscometer. The viscosity and shear stress was recorded by varying the shear rate [21].

\section{Conductivity measurements}

The conductivity of the selected formulations was done to observe the type of microemulsion formed. The conductivity of the formulations was analysed with a conductivity meter, using conductivity cells with a cell constant of 1.0 consisting of two platinum plates separated by desired distance and liquid placed between the platinum plates acting as a conductor.

\section{Thermoreversible microemulsion}

\section{Sol-gel transition temperature and gelling time}

For in situ gel forming systems, the sol-gel transition temperature and time should be determined. Gelling time is the time required for first detection of gelation of in situ gelling system. Thermosensitive in situ gel should be checked for in situ gelling at body temperature.

\section{Drug content}

The accurately weighed amount of prepared gel was taken into a 10 $\mathrm{ml}$ volumetric flask and dissolved in methanol. Suitable dilution was made and absorbance was checked at $230 \mathrm{~nm}$ in triplicate to determine the actual drug concentration in the formulation, using uv spectrophotometer.

\section{Entrapment efficiency}

Entrapment efficiency of mucoadhesive microemulsion was determined by the centrifugation method. Mucoadhesive microemulsion (containing an equivalent to $50 \mathrm{mg}$ of drug) was centrifuged at $20000 \mathrm{rpm}$ for $1 \mathrm{~h}$ in a refrigerated centrifuge to collect the supernatant liquid. The collected liquid was filtered to measure the free drug concentration after suitable dilution with methanol. The absorbance was measured at $230 \mathrm{~nm}$ in a uv spectrophotometer to calculate the entrapment efficiency using the following formula:

$$
\text { Entrapment efficiency \% }=\frac{\text { Intial amount of durg in ME }- \text { Free drug }}{\text { Intial amount of durg in ME }} \times 100
$$

\section{PH}

The $\mathrm{pH}$ of the prepared in-situ gels was determined to evaluate possible irritation effects on the mucosa. The $\mathrm{pH}$ of the gel was measured at room temperature by directly immersing the $\mathrm{pH}$ electrode into the samples [22].

\section{Rheological studies}

The gelled systems were evaluated for their rheological behaviour using Anton Paar Rheometer (Rheo QC), a cup and bob viscometer. The viscosity was determined at various shear rates by subjecting the gel to different torque values. 


\section{Mucoadhesion strength}

The mucoadhesive strength of formulations under investigation was evaluated by measuring the force required to detach the formulation from a mucin disc using Texture Analyzer ${ }^{\mathrm{TM}}$ (Stable Micro Systems TA-XT. plus Texture Analyzer Godalming, U. K. Mucin discs were prepared by compression of a known weight of mucin $(250 \mathrm{mg})$ using a hydraulic press with a diameter of $20 \mathrm{~mm}$. Prior to mucoadhesion testing, the mucin disc was hydrated by submersion in $5 \%$ solution of mucin for 30 s. The excess surface liquid was removed by gentle blotting and they were attached to the lower end of the probe. The in-situ gels were packed into the lower platform of the texture analyzer. The probe holding the mucin disc was lowered on to the surface of the gel with a constant speed of $0.5 \mathrm{~mm} / \mathrm{sec}$ and a contact force of $10 \mathrm{~g}$ was applied. After keeping in contact surfaces for 120 s, the probe was then moved vertically upward at a constant speed of $0.1 \mathrm{~mm} / \mathrm{sec}$. Maximum detachment force was obtained from the force-distance graph. From the force-distance plot, the area under the curve was calculated as mucoadhesion. The tests were conducted at $37{ }^{\circ} \mathrm{C}$ and each experiment was carried out in triplicate.

\section{In vitro drug release}

To determine the release behaviour of the drug from the thermoreversible formulation, the in vitro release experiment was carried out using Franz diffusion cell including a donor chamber and an acceptor compartment. A dialysis membrane (MWCO 800014000) with a constant contact area of $3.14 \mathrm{~cm}^{2}$ was fixed between the donor and receptor chamber. $1 \mathrm{ml}$ of the formulation was added to the donor chamber. The receptor compartment was filled with 30 $\mathrm{ml}$ of mixture medium of phosphate buffer ( $\mathrm{pH}$ 6.4). The receptor solution was subjected to magnetic stirring at 200 RPM and kept at $37{ }^{\circ} \mathrm{C}$ in order to mimic in vivo conditions. At predetermined time intervals such as $0 \mathrm{~h}, 0.5 \mathrm{~h}, 1 \mathrm{~h}, 2 \mathrm{~h}, 3 \mathrm{~h}, 4 \mathrm{~h}, 6 \mathrm{~h}, 8 \mathrm{~h}, 10 \mathrm{~h}, 12 \mathrm{~h}$ and $24 \mathrm{~h}, 1 \mathrm{ml}$ of receptor solution was withdrawn and $1 \mathrm{ml}$ of blank receptor solution was added to maintain a constant volume. The samples were centrifuged at $10,000 \mathrm{rpm}$ for $20 \mathrm{~min}$ and the supernatant was further diluted with methanol for the analysis of dlx-hcl by uv [23].

$$
\text { CR (\%) }=\frac{\text { Cumulative release amount of drug }}{\text { Total amount of drug in formulation }}
$$

\section{Ex vivo drug permeation studies}

The ex-vivo diffusion study was performed using goat nasal mucosa, collected from a slaughterhouse. The nasal conchae was collected in phosphate buffer pH 6.4 and washed three times with phosphate buffer pH6.4. The extraneous tissues were removed with a surgical blade so as to separate out the mucosa. The prepared nasal mucosa was mounted on a Franz diffusion cell with the dermal side facing towards the receptor compartment. Stabilization was performed by stirring the receiving medium with a magnetic stirrer for $30 \mathrm{~min}$ in phosphate buffer $\mathrm{pH}$ 6.4. After stabilization, phosphate buffer was replaced with fresh media (phosphate buffer $\mathrm{pH}$ 6.4) and ex-vivo diffusion study was performed at a temperature maintained at 37 ${ }^{\circ} \mathrm{C} \pm 0.5 .1 \mathrm{ml}$ formulation was placed on the donor side of diffusion cell (area $=3.14 \mathrm{~cm}^{2}$ ). $1 \mathrm{ml}$ of sample was withdrawn at time intervals of $0 \mathrm{~h}, 0.5 \mathrm{~h}, 1 \mathrm{~h}, 2 \mathrm{~h}, 3 \mathrm{~h}, 4 \mathrm{~h}, 6 \mathrm{~h}, 8 \mathrm{~h}, 10 \mathrm{~h}, 12 \mathrm{~h}$ and $24 \mathrm{~h}$ and replaced with an equal volume of the phosphate buffer $\mathrm{pH} 6.4$ and assayed. Graph was plotted between \% cumulative drugs released versus time. Slopes of the linear portion of the graph were used to calculate the permeation flux.

\section{Nasal ciliotoxicity studies}

Nasal mucosa of sheep was collected from the slaughterhouse in saline phosphate buffer ( $\mathrm{pH}$ 6.4). The membrane was placed in $10 \%$ formalin solution to stabilize the nasal membrane. Four pieces of sheep nasal mucosa (P1, P2, P3 and P4) of identical thickness were taken and mounted on the glass slide. P1 was treated with $0.5 \mathrm{ml}$ of phosphate buffer $\mathrm{pH} 6.4$ (negative control), $\mathrm{P} 2$ treated with $0.5 \mathrm{ml}$ of isopropyl alcohol (positive control), P3 and P4 were treated with formulations selected from the ex vivo studies. The mucosa was subjected to histological studies to evaluate the nasal ciliotoxicity of the formulations. The specimens were visualised though photomicrograph at $100 \mathrm{x}$ magnification [24].

\section{Pharmacodynamic studies}

Animals: Female wistar rats bred in Central Animal House facility of Panjab University, Chandigarh, and weighing 140-270 kg were used. The animals were housed under standard light/dark cycle with food and water provided ad libitum. Animals were acclimatized to laboratory condition before the test. The experiments were performed between 9.00 to $17.00 \mathrm{~h}$. The experimental protocol was approved by the Institutional Ethics Committee of Institute (IAEC/589). Rats were weighed and divided into groups as follows:

- The first group (N) of six animals comprised of naïve control rats which were neither exposed to stressors nor given any treatment.

- Second group (P) comprised of positive control rats which were not given any treatment; however, they were exposed to stress conditions.

- The third group (F1) comprised of rats which were given intranasal formulation F1

- The fourth group (F2) comprised of rats administered with intranasal formulation F2

- The fifth group (FD) comprised of rats which were given a free drug suspension, orally.

Evaluation and comparison of the antidepressant effect of the developed in situ gelling microemulsions were performed using chronic unpredictable mild stress (cums) model of depression [25].

\section{Locomotor activity test}

The locomotor activity in test rats was analyzed using Actophotometer which recorded (digital) movements of the animal inside the test chamber. This test provides an index of basal locomotor activity in a familiar environment. The rats were placed individually inside the chamber of actophotometer for $10 \mathrm{~min}$. Average locomotor scores of various groups were compared with each other [26].

\section{Sucrose preference test}

The sucrose preference test was employed herein to determine anhedonia, one of the core symptoms of major depression in humans. This test was performed at the end of $3 \mathrm{w}$ of cums exposure. Briefly, before the test mice were trained to adapt to a sucrose solution $(1 \%$ $\mathrm{w} / \mathrm{v}$ ): two bottles of sucrose solution were placed in each cage for $24 \mathrm{~h}$ and then 1 bottle of sucrose solution was replaced with water for $24 \mathrm{~h}$. After adaption, the rats were deprived of food and water for $24 \mathrm{~h}$. Sucrose preference test was conducted by placing a bottle containing $100 \mathrm{ml}$ of water and another bottle containing $1 \%$ sucrose solution in the animal cages for subsequent $24 \mathrm{~h}$ period. The volumes consumed from each of these bottles were recorded and the sucrose preference (\%) was calculated [27].

$$
\text { Sucrose preference } \%=\frac{\text { sucrose consumption }}{(\text { sucrose consumption }+ \text { water consumption) }} \times 100
$$

\section{Forced swim test}

Rats were individually forced to swim inside a rectangular glass jar $\left(25 \times 12 \times 25 \mathrm{~cm}^{3}\right)$ containing $15 \mathrm{~cm}$ of water maintained at $23-25$ ${ }^{\circ} \mathrm{C}$. After the initial 2-3 min of vigorous activity, the animals showed a period of immobility by floating with minimum movements. The animal was considered to be in an immobile state when it remains floating passively in the water in a slightly hunched but upright position with its nose above the water surface. The total immobility period of 4 min after 2 min of habituation was recorded with the help of a stopwatch and averages of immobility time for various groups were compared using a bar graph [28].

\section{Biochemical estimation}

\section{Preparation of tissue homogenate}

At the end of $27^{\text {th }} d$, the rats were sacrificed by cervical dislocation. Brains of the animals were isolated and rinsed in ice-cold phosphate buffer saline (pH6.4). Hippocampus regions of the brain were isolated 
and homogenized in phosphate buffer saline $(\mathrm{pH} 6.4)$ to make a $10 \%$ homogenate. The $10 \%$ homogenate was then centrifuged at $10,000 \mathrm{rpm}$ at $4{ }^{\circ} \mathrm{C}$ for $10 \mathrm{~min}$ and supernatants were collected from the hippocampus brain homogenate (hbh) and used for various biochemical estimations.

\section{Collection and preparation of plasma samples}

Anaesthetized mouse was held in the hand with its head pointing down. The tip of a fine capillary was inserted into the corner of the eye socket underneath the eyeball, directing the tip at 45-degree angle toward the middle of the eye socket. The capillary was rotated between fingers during the forward passage. Gentle downward pressure was applied and then released until the vein was ruptured and blood entered the capillary and was collected suitably.

\section{Estimation of protein}

The protein content was measured by biuret method using bovine serum albumin (bsa) as standard [29]. $0.1 \mathrm{ml}$ of hbh, $2.9 \mathrm{ml}$ of sodium chloride, $3 \mathrm{ml}$ of working buiret reagent were mixed together. (Biuret reagent was prepared by mixing $4.5 \mathrm{~g}$ of sodium potassium tartrate in $40 \mathrm{ml}$ of $2.0 \mathrm{~N} \mathrm{NaOH}$ with $1.5 \mathrm{~g}$ copper sulphate. To this solution, potassium iodide was added and total volume was made upto $100 \mathrm{ml}$ with $0.2 \mathrm{~N}$ of $\mathrm{NaOH}$ ). This mixture was kept at room temperature for $10 \mathrm{~min}$ and its absorbance was measured at $540 \mathrm{~nm}$.

\section{Estimation of reduced glutathione}

$1 \mathrm{ml}$ of hbh was precipitated with $1 \mathrm{ml}$ of $4 \%$ sulfosalicylic acid and then these samples were incubated at $4{ }^{\circ} \mathrm{C}$ for $1 \mathrm{~h}$. The samples were centrifuged at $1,200 \times \mathrm{g}$ for $15 \mathrm{~min}$ at $4{ }^{\circ} \mathrm{C}$. To $1 \mathrm{ml}$ of this supernatant, $2.7 \mathrm{ml}$ of phosphate buffer $(0.1 \mathrm{M}, p \mathrm{H}$ 8) and $0.2 \mathrm{ml}$ of $0.01 \mathrm{M}$ 5,5-dithiobis (2-nitrobenzoic acid) (dtnb) $(4 \mathrm{mg} / \mathrm{ml}$ ) to a total volume of $3 \mathrm{ml}$ were added. The yellow colour developed was read immediately at $412 \mathrm{~nm}$ uv-vis spectrophotometer. Results were calculated using molar extinction coefficient of chomophore $(1.36 \times$
$104 \mathrm{M}^{-1} \mathrm{~cm}^{-1}$ ) and expressed as nanomoles of reduced glutathione per milligram protein [30].

\section{Estimation of lipid peroxidation}

The quantitative measurement of lipid peroxidation was performed according to the method of Wills [31]. The amount of malondialdehyde (mda), a measure of lipid peroxidation, was measured by reaction with thiobarbituric acid at $532 \mathrm{~nm}$ using uv spectrophotometer. The values were calculated using molar extinction coefficient of chomophore $(1.56 \times$ $105 \mathrm{M}-1 \mathrm{~cm}-1$ ) and expressed as a percentage of the control group.

\section{Statistical analysis}

The statistical significance of the results obtained from the release study was analyzed by one way ANOVA using SPSS (Version 17.0) software. A difference below the probability level of $0.05(95 \%$ confidence interval) was considered statistically significant.

\section{Statement of human and animal rights}

This article does not contain any studies with human subjects performed by any of the authors; all institutional and national guidelines for the care and use of laboratory animals were followed. The experimental protocol was approved by the Institutional Animal Ethical Committee (IAEC) constituted under CPCSEA (45/GO/ReBI/S/99/CPCSEA).

\section{RESULTS AND DISCUSSION}

\section{Solubility studies}

The solubility of duloxetine hcl in various oils, surfactants and cosurfactants were measured. Each experiment was performed in triplicate and averages of solubility in various excipients were represented graphically in fig. 1 . Duloxetine showed maximum solubility in capmul mcm as compared to its solubility in other oils, while labrasol and transcutol $p$ had shown higher drug solubility in comparison with other surfactants and cosurfactants respectively which were used in the solubility studies and hence, were used for further studies.

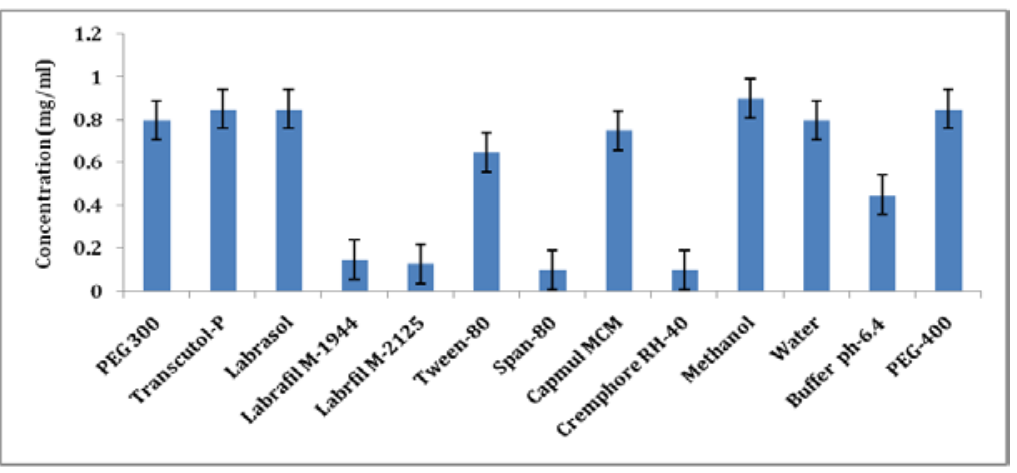

Fig. 1: Solubility data of dlx-hcl in various excipients, (mean $\pm S D, N=3$ )

\section{Pseudoternary phase diagram}

Two pseudoternary phase diagrams (1:1and 2:1) were constructed using capmul $\mathrm{mcm}$ as oily phase and labrasol as surfactant and transcutol $\mathrm{p}$ as cosurfactant fig. 2 (A and B). The microemulsions were selected from the o/w microemulsion region of pseudoternary phase diagrams which were subjected to thermodynamic stability studies including heating-cooling cycle, centrifugation testing and freeze-thaw cycle. The microemulsions which passed these test were selected for further characterization.

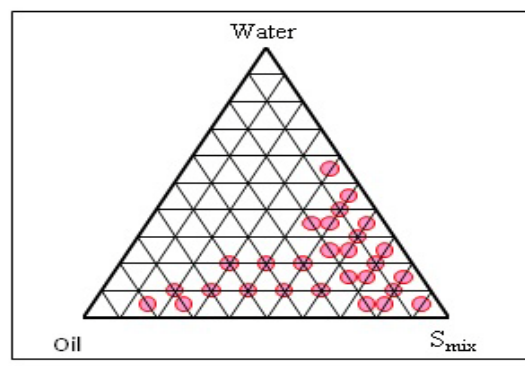

(A)

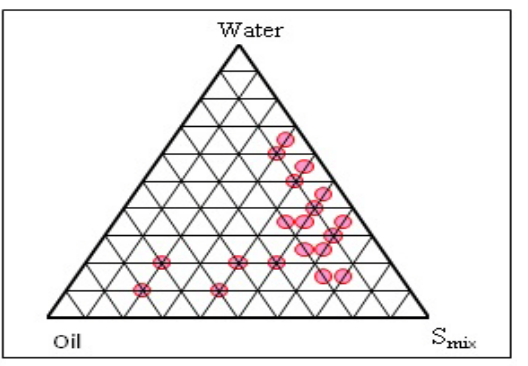

(B)

Fig. 2: Pseudo-ternary phase diagram with different Smix ratio i.e., (A) 1:1 and (B) 2:1. (N=3) 


\section{Preparation of formulation}

Intranasal thermoreversible gelling microemulsion formulations were prepared using chitosan as controlled release, mucoadhesive polymer and absorption enhancer and PF127 as a gelling agent. ME 1, ME 2, ME

3 formulations were successfully prepared with variation in the concentration of PF127 ranging from 20-23\%. Prepared thermoreversible gelling microemulsion formulations were evaluated for physical properties like particle size,zeta potential, viscosity, conductivity, sol-gel transition temperature and gelling time, drug content, entrapment efficiency, $\mathrm{pH}$, mucoadhesion, in vitro release studies, ex vivo permeation, pharmacodynamic studies.

Table 1: Compositions of various intranasal duloxetine hydrochloride thermoreversible microemulsions

\begin{tabular}{llllll}
\hline S. No. & Batch No. & Capmul mom (\%) & $\begin{array}{l}\text { Labrasol: Transcutol P } \\
\text { S mix (\%) }^{(\%)}\end{array}$ & $\begin{array}{l}\text { Water } \\
\text { (\%) }\end{array}$ & $\begin{array}{l}\text { Pluronic F127 (\%) } \\
\text { (\%) }\end{array}$ \\
\hline 1 & ME 1 & 5 & $50(2: 1)$ & 45 & 20 \\
2 & ME 2 & 15 & $50(2: 1)$ & 35 & 20 \\
3 & ME 3 & 15 & $50(2: 1)$ & 35 & 23 \\
\hline
\end{tabular}

$\mathrm{S}_{\text {mix }}$ : Surfactant mix, Capmul mcm: Capmul medium chain mono-diglyceride (mean $\pm \mathrm{SD}, \mathrm{N}=3$ )

\section{Characterization of formulation}

The average diameter of particles in the selected formulation was in the range of $100 \mathrm{~nm}$ to $190 \mathrm{~nm}$. The zeta potential of the microemulsion formulation was found to be in the range of-
35.45 to- $55.32 \mathrm{mV}$. These negative zeta potential values indicate stability of the microemulsion due to its double-layer repulsion between droplets. The conductivity measurements were in the range of $0.105-0.128$ concluding that the formulations were of o/w type.

Table 2: In situ gelling time and gelling temperature for formulation ME1, ME2 and ME3

\begin{tabular}{lll}
\hline Formulations & Mean gelling time \pm SD $^{*}$ & Mean gelling temperature \pm SD \\
\hline ME 1 & $5.67 \pm 0.51$ & $28^{\circ} \mathrm{C} \pm 0.34$ \\
ME 2 & $6.33 \pm 0.31$ & $29^{\circ} \mathrm{C} \pm 0.23$ \\
ME 3 & $6.89 \pm 0.72$ & $32{ }^{\circ} \mathrm{C} \pm 0.43$ \\
\hline
\end{tabular}

ME: Microemulsion, ${ }^{*}(\operatorname{mean} \pm \mathrm{SD}, \mathrm{N}=3)$

Table 2 depicted the in situ gelling time and gelling temperatures of microemulsion formulations. Upon administration, the formulation undergoes rapid sol to gel transition by ionic gelation. Gellling temperatures ranging from $25{ }^{\circ} \mathrm{C}$ to $37^{\circ} \mathrm{C}$ have been considered to be suitable for thermoreversible formulations. If lower than $25^{\circ} \mathrm{C}$, a gel might be formed at room temperature leading to difficulty in manufacturing and administering whereas, if the gelation temperature is higher than $37^{\circ} \mathrm{C}$, the formulation might exist in its liquid state in the nasal cavity body temperature of $34^{\circ} \mathrm{C}$, resulting in its nasal clearance at an early stage [32]. Accordingly, formulations suitable for intranasal delivery were selected.

Drug content of formulations was found to be in the range of $98.98 \pm 1.40 \%$ to $99.75 \pm 0.58 \%$., of the theoretical value $(10 \mathrm{mg} / \mathrm{ml})$. The $\mathrm{pH}$ of the microemulsion formulations was found to be in the range 5.9 to 6.4 i.e., closer to neutral $\mathrm{pH}$ range of the nasal cavity indicating no irritation to the nasal mucosa. The viscosity of the prepared formulation was found to be in the range of $25.7 \pm 1.6 \mathrm{cp}$ to $55.4 \pm 2.3 \mathrm{cp}$. Rheological study of in-situ gel formulations at $3 \% \mathrm{C}$ showed non-newtonian flow whereas at $9 \mathrm{C}$ formulations showed Newtonian flow due to the presence of pluronics, a non-ionic polypropylene oxide triblock copolymer which aggregates into micelles at $37 \mathrm{C}$. When administered intranasally, the viscosity of the formulation was increased, aiding dispersion in the nasal cavity. Further, mucoadhesion force was measured to showcase a range of $0.259 \mathrm{~N}$ to $0.263 \mathrm{~N}$. The developed dlx-hcl thermoreversible in situ gel, therefore, would be retained in the nasal cavity for prolonged intervals with reduced expectancy to leakage and inconvenience.

\section{In vitro drug release}

In vitro release studies were carried out on Franz-diffusion cell using cellophane membranes as a barrier of the selected formulations i.e. ME1, ME2, and ME3. The cumulative percentage drug release at different time intervals of different formulations are shown in fig. 3. Data obtained from in vitro release experiments depicted that drug released from the formulation in a controlled manner and exhibiting sustained release.

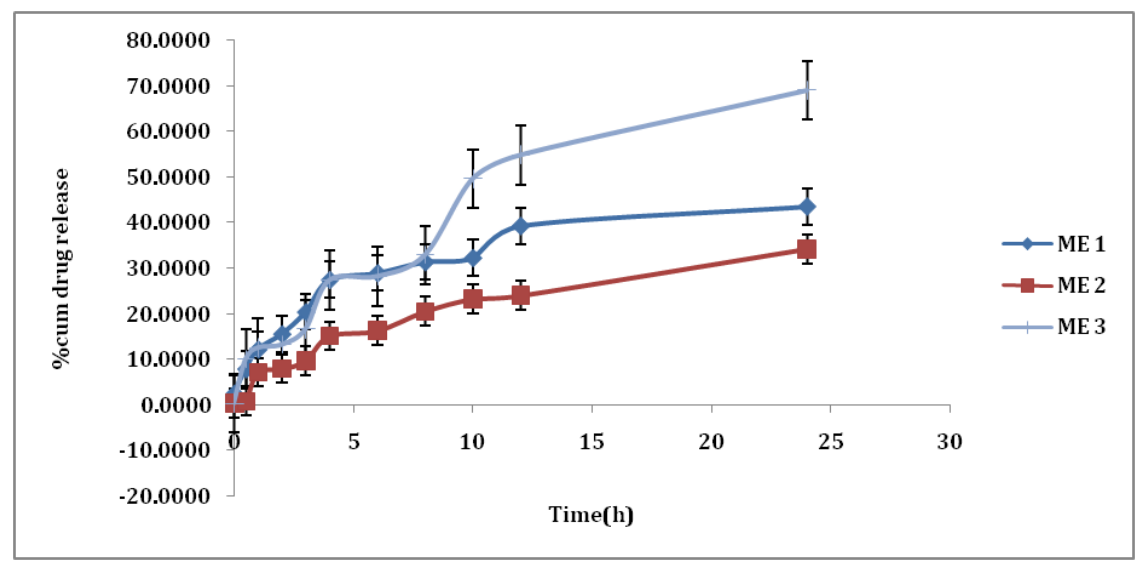

Fig. 3: Comparison of cumulative percentage drug release profiles, $($ mean $\pm S D, N=3)$ 


\section{Ex vivo drug permeation}

The study depicted release potential of the gel, the pattern of drug release through in-situ gels were explained by Higuchi model which clearly represents controlled drug delivery in fig. 4 . The selected formulation had shown significant release from the nasal mucosa with sustained effect. Higher flux value found for formulations may be attributed to the presence of chitosan.

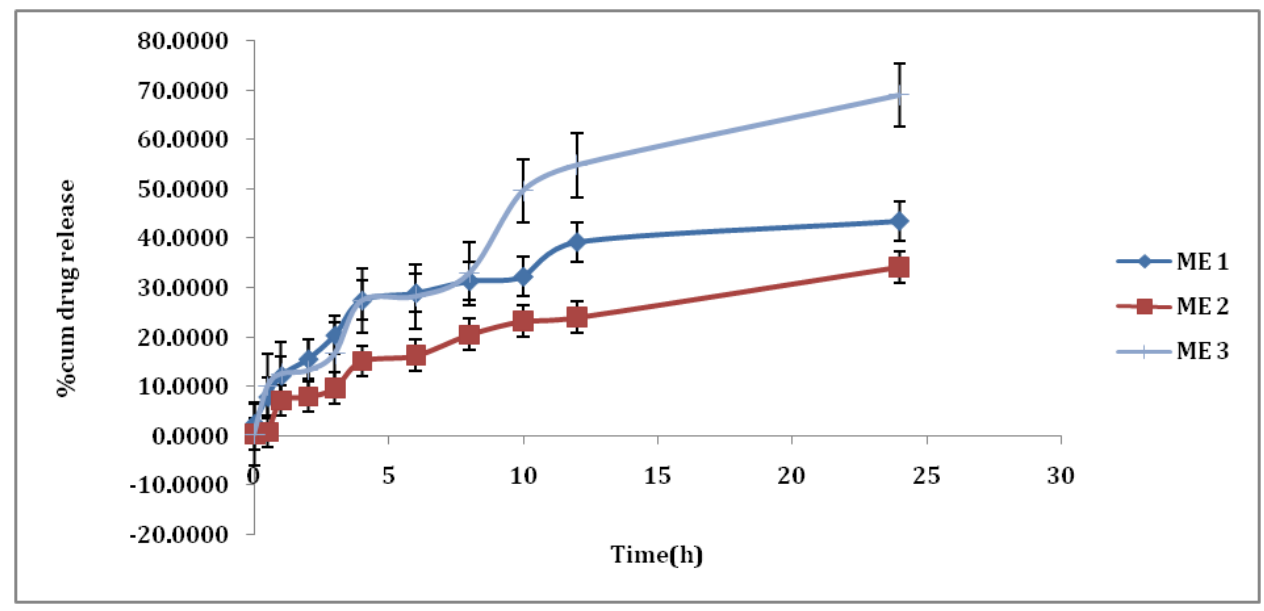

Fig. 4: Permeation of various formulations though nasal mucosa, (mean $\pm \mathrm{SD}, \mathrm{N}=3$ )

\section{Nasal ciliotoxicity studies}

The chemicals and solubility enhancers used in the formulation may cause harm, especially in cases of chronic application, as many of them are irritant in nature. The acceptance criteria for the selection of the oil, surfactant and other excipients, not only depends on its ability to enhance solubility and absorption but also upon its overall safety profile on both local and systemic effect. Thus nasal mucosa toxicity was evaluated to determine any toxicity caused by the

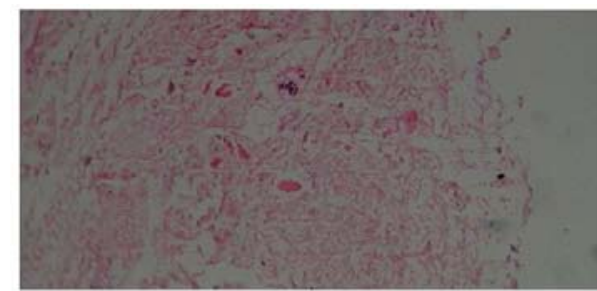

(A)

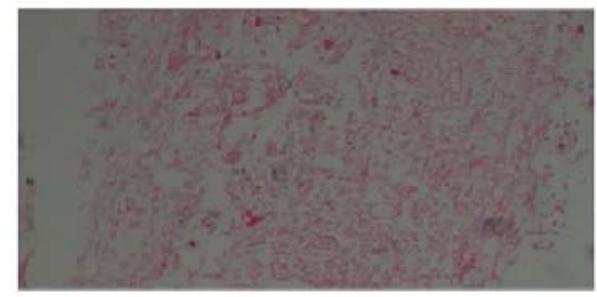

(c) excipients used in the formulation. The intranasal microemulsion treated nasal mucosa showed no signs of vascular congestion and sub-epithelial edema, necrosis or abnormal histopathologic changes. It also showcased negligible irritation to the nasal mucosa, implying this formulation to be of excellent nasal tolerance. The results as shown in fig. 5(A, B and C) revealed that the developed intranasal formulation was non-ciliotoxic and suitable for nasal application whereas, fig. 5(D) revealed severe alterations on the mucosal layer upon treatment with $1 \% \mathrm{v} / \mathrm{v}$ isopropyl alcohol.

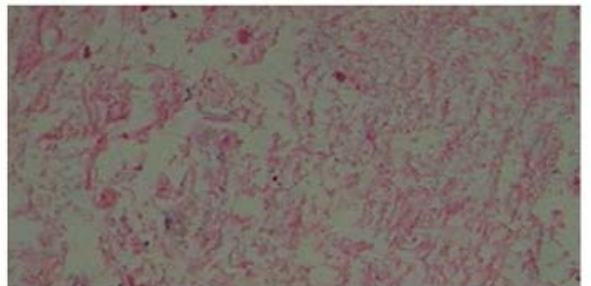

(B)

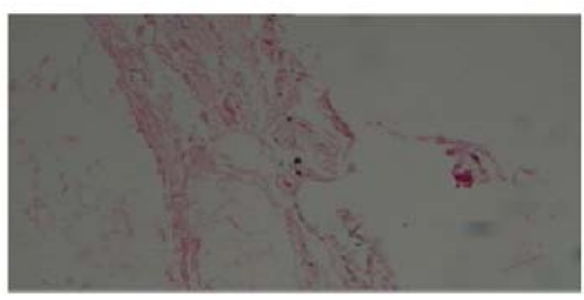

(D)

Fig. 5: Histopathology of (A) Formulation F1 (B) Formulation F2 (C) Nasal membrane treated with phosphate buffer (D) Nasal membrane treated with isopropyl alcohol, $(\mathrm{N}=3)$

\section{Pharmacodynamic studies}

Behavioural characteristics of the animal models and humans are impaired in chronic stress. The present study aimed to evaluate and compare the protective effect of dlx-hcl intranasal formulation in comparison to the free drug suspension given by oral route, as prescribed in depression. Microemulsion ME1 and ME2 mentioned earlier in table 1 were administered intranasally as formulation F1 and F2 respectively. Also, oral suspension, FD of the free drug was administered orally.

\section{Locomotor activity}

In order to detect the association of neurological functions with changes in motor activity, the locomotor activity of rats was tested by digital actophotometer. The locomotor activity of cums control group was significantly decreased after $21 \mathrm{~d}$ of stress in comparison to the naive group of animals. There was a significant $(\mathrm{p}<0.05)$ decrease in the ambulations of the stress-induced mice compared to the naive group. Locomotor activity of the treated group was restored upon with dlx-hcl. Formulation F1 and F2 were given intranasally and oral suspension, FD 
of the free drug was administered orally. The intranasal formulation exhibited an enhanced effect which was $50 \%$ more in comparison to the orally administered free drug, shown in fig. 6(A). This implies that the intranasal formulations were far more effective in comparison to the oral drug suspension.

\section{Sucrose preference test}

The sucrose preference test was employed herein to determine anhedonia, the inability to gain pleasure from normally pleasurable experiences, which is one of the core symptoms of depression. A significant difference in sucrose preference was observed in fig. 6(B) between naive group and control animals $(81 \%$ in the naive group and $39 \%$ in control group sucrose was consumed). Moreover, groups treated intranasally with F1and F2 formulations showed a significantly increased effectiveness with F2 proving to be $55 \%$ more efficient than the oral formulation.

\section{Force swim test}

When forced to swim in the confined space, rats after an initial phase of vigorous activity, cease to struggle, surrendering themselves to the experimental conditions, which showed their helplessness or despair behaviour. This reflects the lowered mood in laboratory animals and could be used for screening of the antidepressant agents [33]. The immobility in this test reflects the development of despair behaviour which disengaged the animal from active forms of coping up with stressful stimuli, and this behaviour is sensitive to antidepressants.

In animals exposed to cums, there was a significant $(\mathrm{p}<0.05)$ increase in the immobility time as compared to the naive group. Animals treated with intranasal formulation F1 and F2 as well as oral marketed formulation, when forced to swim, showed reduction in the immobility time as compared to control animals whereas, formulation F2 completely decreased the immobility time of the rats and the results were similar to those observed for naïve animals with $60 \%$ enhanced effectiveness as compared to the oral free drug suspension as depicted in fig. 6(C). This inferred the intranasal formulations, effectual in comparison to the oral free drug suspension.

\section{Biochemical estimations}

\section{Reduced glutathione}

Reduced glutathione levels are significantly $(\mathrm{p}<0.05)$ decreased in the hbh of stress-induced mice by $90 \%$ compared to unstressed mice. Formulation F1, F2 given by intranasal route and oral free drug suspension administered for 21 successive days, significantly $(p<0.05)$ increased reduced glutathione levels in unstressed rats as compared to the control rats. As seen in fig. 6(D), Formulation F1 and F2 showed significant $(\mathrm{p}<0.05)$ increase in the glutathione levels as compared to oral one, suggesting restoration of reduced glutathione levels on treatment with dlx-hcl given intranasally. Specifically, the F2 intranasal formulation demonstrated a $40 \%$ enhanced treatment with respect to the oral suspension of free drug.

\section{Lipid peroxidation}

In the animals exposed to chonic unpredictable mild stress, there was a significant $(\mathrm{p}<0.05)$ increase in the hippocampal mda levels compared to animals in the naïve group. In the animals treated with intranasal formulations of dlx-hcl, F1 and F2 expressed reduction in the lipid peroxidation levels as demonstrated in fig. 6(E). In comparison to the oral free drug suspension, lipid peroxidation was significantly reduced by $54 \%$ on treatment with the F2 intranasal formulation.

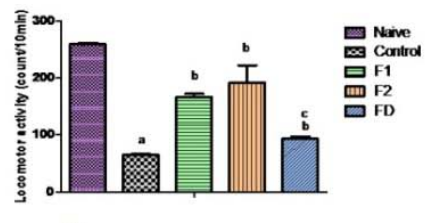

(A)

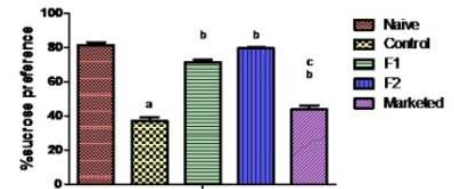

(B)

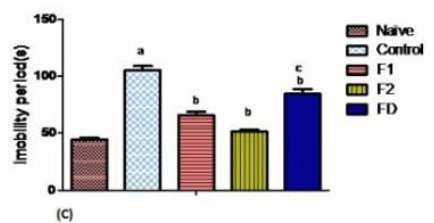

(c)

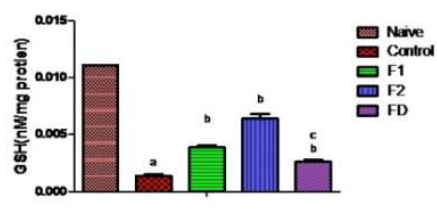

(D)

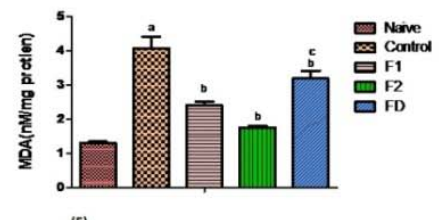

Fig. 6: Effect of intranasal formulation (F1 and F2), oral formulation (FD) on (A) locomotor score (B) sucrose preference (C) immobility time in the FST (D) reduced glutathione levels in the hippocampal region (E) lipid peroxidation in the hippocampal region, of stressinduced rats, (mean $\pm \mathrm{SD}, \mathrm{N}=3$ )

\section{CONCLUSION}

The conclusion derived from this research demonstrated that the thermoreversible microemulsion for intranasal delivery can be employed to improve drug brain levels and increase dlx-hcl bioavailability. A formulation containing $15 \%$ capmul $\mathrm{mcm} 50 \%$ of 2:1 Smix (labrasol: transcutol p), and water along with $20 \%$ pluronic F127 and $0.5 \%$ of chitosan showed better results in the treatment of depression as compared to oral free drug suspension of duloxetine in rats. It also demonstrated sustained release, enhanced in vitro permeability with no evidence of nasal ciliotoxicity. Therefore, enhanced rate and extent of brain permeability following intranasal administration may be favorable in decreasing the dose along with the frequency of dosing. Hence this formulation strategy can be used as an effective targeting technique for the drugs having low bioavailability and poor brain penetration.

\section{ACKNOWLEDGEMENT}

Authors acknowledge the Indian Council of Medical Research (ICMR), New Delhi, India, for funding and granting this project. The authors are also thankful for gift samples from Abitec Corporation (Janesville, United States) and Gattefosse Asia Ltd. (Mumbai, India).

\section{AUTHORS CONTRIBUTIONS}

The corresponding author, Gurpreet Singh had developed the experimental section of the work. Writing up and corrections were carried out by Gurpreet Singh, Kirti Singh and Nisha Rawat. Dr. Amita Sarwal and Prof. VR Sinha co-operated in writing results, data interpretation, and reference management.

\section{CONFLICT OF INTEREST}

The authors declare no conflict of interest. 


\section{REFERENCES}

1. Bisht R. Brain drug delivery system: a comprehensive review on recent experimental and clinical findings. Int J Pharm Sci Res 2011;2:*792-806.

2. Alam MI, Beg S, Samad A, Baboota S, Kohli K, Ali J, et al. Strategy for effective brain drug delivery. Eur J Pharm Sci 2010;40:385-403.

3. Thorne R, Pronk G, Padmanabhan V, Frey Wn. Delivery of insulin-like growth factor-I to the rat brain and spinal cord along olfactory and trigeminal pathways following intranasal administration. Neurosci 2004;127:481-96.

4. Elshafeey $\mathrm{AH}$, Bendas ER, Mohamed $\mathrm{OH}$. The intranasal microemulsion of sildenafil citrate: in vitro evaluation and in vivo pharmacokinetic study in rabbits. AAPS PharmSciTech 2009;10:361-7.

5. Vyas TK, Babbar A, Sharma R, Misra A. Intranasal mucoadhesive microemulsions of zolmitriptan: preliminary studies on brain-targeting. J Drug Target 2005;13:317-24.

6. Shital Butani TS, Kunal Parmar, Amarjitsing Rajput. Development of rizatriptan benzoate microspheres for the nose to brain targeting. Int J Appl Pharm 2016;8:69-74.

7. Pires PC, Santos AO. Nanosystems in nose-to-brain drug delivery: A review of non-clinical brain targeting studies. J Controlled Release 2018;270:89-100.

8. Talegaonkar S, Azeem A, Ahmad FJ, Khar RK, Pathan SA, Khan ZI. Microemulsions: a novel approach to enhanced drug delivery. Recent Pat Drug Delivery Formulation 2008;2:238-57.

9. Lin Y, Shen Q, Katsumi H, Okada N, Fujita T, Jiang X, et al. Effects of Labrasol and other pharmaceutical excipients on the intestinal transport and absorption of rhodamine123, a P-glycoprotein substrate, in rats. Biol Pharm Bull 2007;30:1301-7.

10. Tenjarla S. Microemulsions: an overview and pharmaceutical applications. Crit Rev Ther Drug Carr Sys 1999;16:461-521.

11. Lieberman HA, Rieger MM, Banker GS. Disperse systems. Pharm Dosage Forms; 1998.

12. Dixit GR, MATHUR VB. Microemulsions: a platform for improvement of solubility and dissolution of poorly soluble drugs. Asian J Pharm Clin Res 2015;8:7-17.

13. Jain DS, Bajaj AN, Tiwari N. In vitro-in vivo assessment and comparison of intranasally administered microemulsion formulations of essential oils for a migraine. Int J Curr Pharm Res 2011;3:47-51.

14. Jogani VV, Shah PJ, Mishra P, Mishra AK, Misra AR. Intranasal mucoadhesive microemulsion of tacrine to improve brain targeting. Alzh Dis Assoc Disorders 2008;22:116-24.

15. Kumar M, Misra A, Mishra A, Mishra P, Pathak K. Mucoadhesive nanoemulsion-based intranasal drug delivery system of olanzapine for brain targeting. J Drug Target 2008;16:806-14.

16. Detke MJ, Lu Y, Goldstein DJ, McNamara RK, Demitrack MA. Duloxetine $60 \mathrm{mg}$ once daily dosing versus placebo in the acute treatment of major depression. J Psych Res 2002;36:383-90.

17. Paulzen M, Hiemke C, Gründer G. Plasma levels and cerebrospinal fluid penetration by duloxetine in a patient with a non-fatal overdose during a suicide attempt. Int J Neuropsych 2009;12:1431-2.

18. Lantz R, Gillespie T, Rash T, Kuo F, Skinner M, Kuan H, et al. Metabolism, excretion, and pharmacokinetics of duloxetine in healthy human subjects. Drug Met Disp 2003;31:1142-50.

19. Kilts CD. Potential new drug delivery systems for antidepressants: an overview. J Clin Psych 2003;64:31-3.

20. Shinde RL, Bharkad GP, Devarajan PV. Intranasal microemulsion for the targeted nose to brain delivery in neurocysticercosis: the role of docosahexaenoic acid. Eur J Pharm Biopharm 2015;96:363-79.

21. Moghimipour E, Salimi A, Eftekhari S. Design and characterization of microemulsion systems for naproxen. Adv Pharm Bull 2013;3:63.

22. Karavana SY, Rencber S. A new in-situ gel formulation of itraconazole for vaginal administration. Pharmacol Pharm 2012;3:417-26.

23. Pathak R, Prasad Dash R, Misra M, Nivsarkar M. Role of mucoadhesive polymers in enhancing delivery of nimodipine microemulsion to the brain via the intranasal route. Acta Pharm Sin B 2014;4:151-60.

24. Mahajan HS, Mahajan MS, Nerkar PP, Agrawal A. Nanoemulsion-based intranasal drug delivery system of saquinavir mesylate for brain targeting. Drug Delivery 2014;21:148-54.

25. Jindal A, Mahesh R, Bhatt S. Etazolate, a phosphodiesterase 4 inhibitor reverses chronic unpredictable mild stress-induced depression-like behaviour and brain oxidative damage. Pharmacol Biochem Behavior 2013;105:63-70.

26. Valecha R, Dhingra D. Behavioral and biochemical evidence for the antidepressant-like activity of Celastrus paniculatus seed oil in mice. Basic Clin Neurosci 2016; 7:*49-56.

27. Serchov T, Calker D, Biber K. Sucrose preference test to measure anhedonic behaviour in mice. Bio-protocol 2016;6. Doi:10.21769/BioProtoc.1958.

28. Brenes Sáenz JC, Villagra OR, Fornaguera Trías J. Factor analysis of forced swimming test, sucrose preference test and open field test on enriched, social and isolated reared rats. Behav Brain Res 2006;169:57-65.

29. Gornall AG, Bardawill CJ, David MM. Determination of serum proteins by means of the biuret reaction. J Biol Chem 1949;177:751-66.

30. Ellman GL. A colourimetric method for determining low concentrations of mercaptans. Arch Biochem Biophys 1958;74:443-50.

31. Wills E. Mechanisms of lipid peroxide formation in animal tissues. Biochem J 1966;99:667-76.

32. Majithiya RJ, Ghosh PK, Umrethia ML, Murthy RSR. Thermoreversible-mucoadhesive Gel for nasal delivery of sumatriptan. AAPS PharmSciTech 2006;7:E80-E6.

33. Porsolt RD, Anton G, Blavet N, Jalfre M. Behavioural despair in rats: a new model sensitive to antidepressant treatments. Eur J Pharm 1978;47:379-91. 